\title{
Stochastic and deterministic analysis of a COVID-19 pandemic model under vaccination strategy : Real cases application
}

\section{Adil Ez-Zetouni ( $\nabla$ adilezzetouni@gmail.com )}

Cadi Ayyad University: Universite Cadi Ayyad

\section{Omar Khyar}

Hassan II Mohammedia University Faculty of Science and Technology Mohammedia: Universite Hassan

II Casablanca Faculte des Sciences Techniques Mohammedia

\section{Karam Allali}

Hassan II Mohammedia University Faculty of Science and Technology Mohammedia: Universite Hassan

II Casablanca Faculte des Sciences Techniques Mohammedia

\section{Khadija Akdim}

Cadi Ayyad University: Universite Cadi Ayyad

\section{Mehdi Zahid}

Cadi Ayyad University: Universite Cadi Ayyad

\section{Research Article}

Keywords: SICVS pandemic model, COVID-19, Vaccination strategy, Local stability, Brownian motion, Stochastic disease extinction, Persistence in mean, Numerical simulations, Real cases application

Posted Date: March 7th, 2022

DOI: https://doi.org/10.21203/rs.3.rs-1346960/v1

License: (a) (1) This work is licensed under a Creative Commons Attribution 4.0 International License. Read Full License 


\title{
Stochastic and deterministic analysis of a COVID-19 pandemic model under vaccination strategy : Real cases application
}

\author{
Adil Ez-Zetouni • Omar Khyar • Karam Allali • \\ Khadija Akdim • Mehdi Zahid
}

Received: date / Accepted: date

\begin{abstract}
In this paper, we present a novel SICVS COVID-19 pandemic model incorporating a vaccination strategy and crowding effect. First, we investigate the steady states and proved the local stability of the corresponding deterministic problem. Then we study the proposed stochastic epidemic model; we have considered a random incidence highlighting all the improbable fluctuations that appear in the infection process. Hence, the COVID-19 transmission parameter in the considered model will be stochastically perturbed by the white noise. We show the disease extinction of the pandemic according to the stochastic noise intensity. It was established, in the stochastic study, the existence of a possible extinction of the pandemic even if the base reproduction number is greater than unity. Furthermore we discuss the persistence in mean of the studied infection in terms of the stochastic model parameters. Our numerical simulations confirm the theoretical findings. The proposed model was validated and supported by a comparison, during the second wave of the pandemic in Morocco, between the trajectories of the clinical data of Covid-19 and those which represent our SICVS model. In addition, the impact of vaccination and social distancing strategies on reducing the infection severity were observed.
\end{abstract}

Keywords SICVS pandemic model · COVID-19 · Vaccination strategy · Local stability · Brownian motion · Stochastic disease extinction · Persistence in mean · Numerical simulations · Real cases application.

\footnotetext{
Omar Khyar and Karam Allali

Laboratory of Mathematics and Applications, Faculty of Sciences and Technologies University Hassan II of Casablanca, PO Box 146, Mohammedia, Morocco.

Tel.: +212623152976

E-mail: omarkhyar@hotmail.com

Adil Ez-Zetouni, Khadija Akdim and Mehdi Zahid

Department of Mathematics, Faculty of Sciences and Technology, Cadi Ayyad University, B.P. 549, Marrakesh C.P. 40.000, Morocco.
} 


\section{Introduction}

Humanity is currently living a real nightmare due to COVID-19 which has devastated all parts of the world making it a pandemic. All countries have joined the race to curb the rapid spread of COVID-19 infection and to reduce the growing mortality rate of populations affected. In the literature, many mathematical models have been developed to describe the evolution of infectiouse diseace [1-8]. Some recent works have been inspired from the natural interaction between susceptible, infected and recovered individuals (SIR) in order to study the evolution of COVID-19 pandemic. In this sense, Chen et al. have established a mathematical SIR model in order to study the impact of social distancing on the spread of COVID-19 [9], the time evolution of COVID-19 disease in different countries was depicted via SIR model in [10], and many other recent papers have led mathematical researchers to conclude that SIR models can better describe the dynamics of this pandemic as well as give more information than using only statistical data. Moreover, Volpert et al. [11] have studied the effect of the quarantine strategy. It has been revealed that the quarantine measure is not enough to eradicate the COVID-19 infection and that other additional strategies need to be considered.

Unfortunately, not all infected individuals are usually reported to be infected cases. Therefore, we can remark the emergence of two type of infected individuals that are the real infected and the confirmed infected ones. To describe mathematically this phenomenon, another compartment must be added to the classical SIR model. In [12], the authors distinguish the reported confirmed COVID-19 cases from the other infected individuals and conclude that more strict control and quarantine measures should be applied to have significant reduce of the infection. In the same context, different works have splitted the COVID-19 infected class to two or more compartments and have studied mathematically and numerically the infection dynamics $[13,14]$. As it is well known, vaccination strategy can save millions of lives around the world knowing that most of epidemics have been eradicated by effective vaccines. To fight against the dangerous COVID-19 pandemic, pharmaceutical companies have produced hundred of millions of injectable doses [15]; however the world requires billions as fast as possible to be administrated worldwide. So far, many other control measures against COVID-19 such as social distancing, isolation, lockdown and quarantine have been undertaken [16-21], however those measures remain limited in term of appreciable effectiveness. Consequently, vaccination remains actually the only hope to eradicate the coronavirus and return to normal life since it has always been considered as an important strategy in fighting against many previous infectious diseases. Accordingly, compartmental epidemiological models with vaccination have been widely investigated by many researchers [22-26]. For this matter, vaccination strategy can be modeled by two main approaches where the vaccinated population can be modeled by incorporating another new compartment $[26,28]$, or it can be included implicity in the other existing compartments by means of new parameter representing the effect of vaccination $[22,27]$.

In this study, we will establish a mathematical model describing the dynamical evolution of COVID-19 pandemic with vaccination strategy and taking into account both real and reported infected individuals. For this purpose, we consider an epidemic model consisting of four ordinary differential equations illustrating the interaction between the susceptible $S$, the real infected $I$, the confirmed infected $C$ and the vaccinated individuals $V$.

$$
\left\{\begin{aligned}
\frac{d S}{d t} & =A-\beta(1-\rho) \frac{S I}{1+a I^{2}}+\eta I+\theta C-(\mu+\gamma) S+\varepsilon V \\
\frac{d I}{d t} & =\beta(1-\rho) \frac{S I}{1+a I^{2}}-\left(\mu+\delta_{1}+\eta+\alpha\right) I, \\
\frac{d C}{d t} & =\alpha I-\left(\mu+\delta_{2}+\theta\right) C, \\
\frac{d V}{d t} & =\gamma S-(\mu+\varepsilon) V
\end{aligned}\right.
$$

with

$$
S(0) \geq 0, I(0) \geq 0, C(0) \geq 0 \text { and } V(0) \geq 0 .
$$

Where $A$ is the recruitment rate, $\beta$ is the disease transmission rate, $a$ is the infection saturated rate, $\varepsilon$ is the rate of vaccinated individuals who became susceptible, $\eta$ and $\theta$ are the recovery rates of real infected individuals and the confirmed infected, respectively; $\delta_{1}$ is the COVID-19 induced death rate of real infected individuals, $\alpha$ is the rate of transmission from the class of real infected to the 


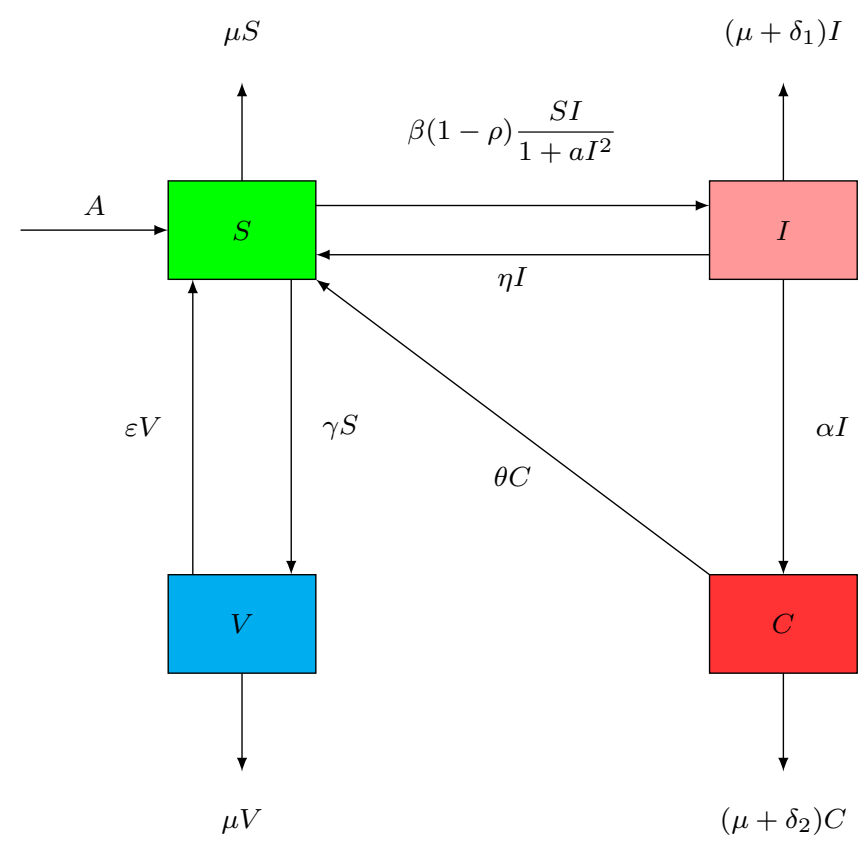

Fig. 1 The flowchart of SICV coronavirus pandemic COVID-19.

class of confirmed infected, $\delta_{2}$ is the COVID-19 induced death rate of confirmed infected individuals, $\gamma$ is the vaccinated susceptible individuals rate and $\mu$ is the natural death rate of the population. Finally, the parameter $\rho$ reflects the efficiency of social distancing. Note that in our model a saturated infection function is taken into consideration in order to illustrate the crowding effect in the studied population.

Nevertheless, random environmental factors such as sudden climate changes, humidity, temperature and also random transmission process of the infection have a considerable impact on the spread of infectious diseases [29-31]. Taking this natural phenomenon into consideration, several works introduced randomness effects into the deterministic biological model in order to reveal the environmental and natural sudden fluctuations of parameters variables in the differential systems. In fact, it is well known that the infection rate is subject to the random fluctuations [32]. Stochastic modeling of diseases by stochastic differential equations is more accurate than mathematical deterministic modeling, because for a fixed initial condition stochastic model can lead to different outputs; whereas a deterministic model will just produce a single result $[31,32]$. In addition, stochastic compartmental model allows to predict the different possibility of the infection evolution and then to take the necessary precautions to avoid some critical epidemic situations [33-37]. Accordingly, in the last years, stochastic perturbed epidemic models have emerged as interesting topics in mathematical epidemiology [38]. Many authors have shown great interest in stochastic models with vaccination strategy [39-42]. Moreover, stochastic COVID-19 epidemic models have been extensively studied by many authors [43-45]. For instance, Boukanjime et al. [43] studied a stochastic COVID-19 epidemic model by incorporating a general incidence rate. The authors investigated the persistence and the extinction of the epidemic in terms of a stochastic threshold. Rihan et al. [46] analyzed a stochastic epidemic COVID-19 model, with cross-immune class and time-delay to study the spread of the infection under random environmental factors such as precipitation, humidity and temperature. The results show that white noise can play an important role in controlling the spread of the coronavirus disease.

In this paper, we will consider that the random fluctuations will be manifested in the infection process. Hence, the COVID-19 transmission parameter $\beta$ in the model (1) will be stochastically perturbed by white noise

$$
\tilde{\beta} d t=\beta d t+\xi d W_{t},
$$

where $W_{t}$ is standard Brownian motion with the property $W_{0}=0$, the volatility parameter $\xi \in \mathbb{R}$ and defined on a complete probability space $(\Omega, \mathcal{F}, \mathbb{P})$ with the filtration $\left(\mathcal{F}_{t}\right)_{t \geq 0}$, satisfying the usual conditions, i.e., it is right continuous and $\mathcal{F}_{0}$ contains all $\mathbb{P}$-null sets. The resulting system of equations 
can be described by the following stochastic model

$$
\left\{\begin{aligned}
d S(t) & =\left(A-\beta(1-\rho) \frac{S I}{1+a I^{2}}+\eta I+\theta C-(\mu+\gamma) S+\varepsilon V\right) d t-\xi \frac{S I}{1+a I^{2}} d W_{t} \\
d I(t) & =\left(\beta(1-\rho) \frac{S I}{1+a I^{2}}-\left(\mu+\delta_{1}+\eta+\alpha\right) I\right) d t+\xi \frac{S I}{1+a I^{2}} d W_{t} \\
d C(t) & =\left(\alpha I-\left(\mu+\delta_{2}+\theta\right) C\right) d t \\
d V(t) & =(\gamma S-(\mu+\varepsilon) V) d t
\end{aligned}\right.
$$

Our paper is organized as follows. In Section 2, we will give the equilibria of model (1), calculate the basic reproduction number and perform the local stability results. In Section 3, we will fulfill the stochastic analysis of model (2) by proving the extinction and persistence in mean of the studied disease. Section 4 is devoted to illustrate our theoretical findings by numerical simulations, we will give also a comparison between the model results and COVID-19 clinical data. The last section concludes our work.

\section{Steady states and local stability}

\subsection{Basic reproduction number}

This subsection is devoted to calculate the basic reproduction number $R_{0}$ [48] associated to model (1) using the next generation matrix. Let $F$ be the nonnegative matrix of new infection cases, and $\mathcal{V}$ the matrix of the transition of infections associated to the model (1). Then we have

$$
F=\left(\begin{array}{cc}
\beta(1-\rho) S_{0} & 0 \\
0 & 0
\end{array}\right), \quad \mathcal{V}=\left(\begin{array}{cc}
\delta_{1}+\mu+\alpha+\eta & 0 \\
-\alpha & \delta_{2}+\mu+\theta
\end{array}\right)
$$

So,

$$
F \mathcal{V}^{-1}=\left(\begin{array}{cc}
\frac{\beta(1-\rho) S_{0}}{\delta_{1}+\mu+\alpha+\eta} & 0 \\
0 & 0
\end{array}\right)
$$

with $S_{0}=\frac{A(\mu+\varepsilon)}{\mu(\mu+\varepsilon+\gamma)}$. The basic reproduction number is the spectral radius of the matrix $F \mathcal{V}^{-1}$. This fact implies that

$$
R_{0}=\frac{A \beta(1-\rho)(\mu+\varepsilon)}{\mu(\mu+\varepsilon+\gamma)\left(\delta_{1}+\mu+\alpha+\eta\right)}
$$

\subsection{The problem steady states}

The steady states of our studied problem (1) are illustrated by the following theorem.

Theorem 1. The model (1) has a disease-free equilibrium $E_{f}$ and an endemic equilibrium $E_{1}$.

Proof To find the steady states of the system (1), we solve the following system

$$
\begin{aligned}
A-\beta(1-\rho) \frac{S I}{1+a I^{2}}+\varepsilon V+\theta C+\eta I-(\mu+\gamma) S & =0 \\
\beta(1-\rho) \frac{S I}{1+a I^{2}}-\delta_{1} I-(\mu+\alpha+\eta) I & =0 \\
\alpha I-\delta_{2} C-(\mu+\theta) C & =0 \\
\gamma S-(\mu+\varepsilon) V & =0 .
\end{aligned}
$$

After a simple resolution, we obtain

- When $I=0$ we find the disease-free equilibrium

$$
E_{f}=\left(\frac{A(\mu+\varepsilon)}{\mu(\mu+\varepsilon+\gamma)}, 0,0, \frac{A \gamma}{\mu(\mu+\varepsilon+\gamma)}\right)
$$


- When $I \neq 0$ we find the endemic equilibrium defined as follows $E_{1}=\left(S^{*}, I^{*}, C^{*}, V^{*}\right)$, where

$$
\begin{gathered}
S^{*}=\frac{S_{0}}{R_{0}}\left(1+a I^{*^{2}}\right), \\
I^{*}=\frac{2\left(R_{0}-1\right)}{\sqrt{\left(\Gamma R_{0}\right)^{2}+4 a\left(R_{0}-1\right)}+\Gamma R_{0}}, \\
C^{*}=\frac{\alpha}{\delta_{2}+\mu+\theta} I^{*}, \\
V^{*}=\frac{\gamma S_{0}}{(\mu+\varepsilon) R_{0}}\left(1+a I^{*^{2}}\right),
\end{gathered}
$$

with $\quad \Gamma=\frac{(\mu+\varepsilon)\left(\delta_{2}+\mu+\theta\right)+\alpha\left(\delta_{2}+\mu\right)}{A\left(\delta_{2}+\mu+\theta\right)}$ and $\quad S_{0}=\frac{A(\mu+\varepsilon)}{\mu(\mu+\varepsilon+\gamma)}$.

It's clear that $E_{1}$ is well defined when $R_{0}>1$.

2.3 Local stability of the equilibria

\subsubsection{Local stability of the disease-free equilibrium}

The local stability of the disease-free equilibrium point $E_{f}=\left(\frac{A(\mu+\varepsilon)}{\mu(\mu+\varepsilon+\gamma)}, 0,0, \frac{A \gamma}{\mu(\mu+\varepsilon+\gamma)}\right)$, is given by the following result:

Proposition 1. When $R_{0}<1$, then the disease-free equilibrium, $E_{f}$, is locally asymptotically stable.

Proof. The Jacobian matrix of the system (1) at $E_{f}$ is given by:

$$
J_{E_{f}}=\left(\begin{array}{cccc}
-(\mu+\gamma) & -\beta(1-\rho) S_{0}+\eta & \theta & \varepsilon \\
0 & \beta(1-\rho) S_{0}-\left(\delta_{1}+\mu+\alpha+\eta\right) & 0 & 0 \\
0 & \alpha & -\left(\delta_{2}+\mu+\theta\right) & 0 \\
\gamma & 0 & 0 & -(\mu+\varepsilon)
\end{array}\right) .
$$

The characteristic polynomial of $J_{E_{f}}$ is

$$
P_{E_{f}}(\lambda)=[\lambda+\mu][\lambda+(\mu+\gamma+\varepsilon)]\left[\lambda+\left(\delta_{2}+\mu+\theta\right)\right]\left[\lambda+\left(\delta_{1}+\mu+\alpha+\eta\right)\left(1-R_{0}\right)\right] .
$$

Therefore, the eigenvalues of $J\left(E_{f}\right)$ are given as follow,

$$
\begin{aligned}
& \lambda_{1}=-\mu<0, \\
& \lambda_{2}=-(\mu+\gamma+\varepsilon)<0, \\
& \lambda_{3}=-\left(\delta_{2}+\mu+\theta\right)<0, \\
& \lambda_{4}=-\left(\delta_{1}+\mu+\alpha+\eta\right)\left(1-R_{0}\right) .
\end{aligned}
$$

Clearly, $\lambda_{1}, \lambda_{2}$ and $\lambda_{3}$ are negative. However, $\lambda_{4}$ is negative when $R_{0}<1$. Consequently $E_{f}$ is locally asymptotically stable when $R_{0}<1$.

\subsubsection{Local stability of the endemic equilibrium}

The local stability of the endemic equilibrium point $E_{1}=\left(S^{*}, I^{*}, C^{*}, V^{*}\right)$, is given by the following result:

Proposition 2. When $R_{0}>1$ then the endemic equilibrium point $E_{1}$ is locally asymptotically stable. 
Proof. The Jacobian matrix of the system (1) at $E_{1}$ is given by:

$$
J_{E_{1}}=\left(\begin{array}{cccc}
\frac{-\beta(1-\rho) I}{1+a I^{*^{2}}}-(\mu+\gamma) & \frac{-\beta(1-\rho) S\left(1-a I^{*^{2}}\right)}{1+a I^{*^{2}}}+\eta & \theta & \varepsilon \\
\frac{-\beta(1-\rho) I}{1+a I^{*^{2}}} & \frac{\beta(1-\rho) S\left(1-a I^{*^{2}}\right)}{1+a I^{*^{2}}}-\left(\delta_{1}+\mu+\alpha+\eta\right) & 0 & 0 \\
0 & \alpha & -\left(\delta_{2}+\mu+\theta\right) & 0 \\
\gamma & 0 & 0 & -(\mu+\varepsilon)
\end{array}\right) .
$$

The characteristic polynomial of $J_{E_{1}}$ is

$$
P_{E_{1}}(\lambda)=(\mu+\varepsilon+\lambda)\left(\lambda^{3}+A_{1} \lambda^{2}+A_{2} \lambda+A_{3}\right)
$$

such that

$$
\begin{aligned}
& A_{1}=a Z I^{*^{2}}+\mu+\delta_{2}+\theta+\frac{\Lambda(\mu+\varepsilon)}{S_{0}} \\
& A_{2}=a\left(\mu+\delta_{2}+\theta\right) Z I^{*^{2}}+\frac{\Lambda(\mu+\varepsilon)}{S_{0}}\left(a Z I^{*^{2}}+\mu+\delta_{2}+\theta\right)+\frac{\beta(1-\rho)\left(\mu+\delta_{1}+\alpha\right)(\mu+\varepsilon) I^{*} S_{0}}{R_{0} S^{*}}
\end{aligned}
$$

and

$$
A_{3}=\frac{a \Lambda(\mu+\varepsilon)\left(\mu+\delta_{2}+\theta\right) Z I^{*^{2}}}{S_{0}}+\frac{\beta(1-\rho) I^{*} S_{0}}{R_{0} S^{*}}\left(\mu+\delta_{1}+\alpha+(\mu+\varepsilon)\left(\mu+\delta_{2}+\theta\right)-\alpha \theta(\mu+\varepsilon)\right),
$$

where $Z=\frac{\beta(1-\rho) S^{*}}{1+a I^{*^{2}}}$. The first eigenvalue of $(4)$ is $\lambda_{1}=-(\mu+\varepsilon)<0$, also it is easy to verify that $A_{1}>0, A_{1} A_{2}-A_{3}>$ and $A_{3}>0$ if $R_{0}>1$ then by using the Routh-Hurwitz Theorem, the other eigenvalues of (4) have negative real parts. Consequently, $E_{1}$ is locally asymptotically stable when $R_{0}>1$.

\section{Stochastic analysis of the model}

3.1 Existence and uniqueness of the global positive solution

Theorem 3. System (2) has a unique positive solution $(S(t), I(t), C(t), V(t))$ on $t \geq 0$, and the solution will remain in $\mathbb{R}_{+}^{4}$ for any given nonnegative initial condition a.s (almost surely).

Proof It is clear that the drift and diffusion components of the stochastic system (2) are locally Lipschitz. According to the theory of stochastic differential equations (SDEs), for any given initial value $(S(0), I(0), C(0), V(0)) \in \mathbb{R}_{+}^{4}$, there exists a unique local solution $(S(t), I(t), C(t), V(t))$ for $t \in\left[0, \tau_{e}\right)$, where $\tau_{e}$ is the explosion time. To show the global existence of this solution, we simply need to show that $\tau_{e}=\infty$ a.s.. Indeed, let $p_{0} \geq 1$ be sufficiently large such that $S(0), I(0), C(0)$ and $V(0)$, all lie within the interval $\left[\frac{1}{p_{0}}, p_{0}\right]$. For each integer $p \geq p_{0}$, define the stopping time $\tau_{p}$ by

$$
\begin{aligned}
\tau_{p}= & \inf \left\{t \in\left[0, \tau_{e}\right), S(t) \notin\left(\frac{1}{p}, p\right) \text { or } I(t) \notin\left(\frac{1}{p}, p\right) \text { or } C(t) \notin\left(\frac{1}{p}, p\right)\right. \\
& \text { or } \left.V(t) \notin\left(\frac{1}{p}, p\right)\right\},
\end{aligned}
$$

where $\inf \emptyset=\infty$ ( $\emptyset$ denotes the empty set). Obviously, $\tau_{p}$ is increasing as $p \rightarrow \infty$. Let $\tau_{\infty}=\lim _{p \rightarrow \infty} \tau_{p}$, whence $\tau_{\infty} \leq \tau_{e}$ a.s. Assume contrarily that $\tau_{e}<\infty$ a.s., then there exists a pair of constants $T>0$ and $\epsilon \in(0,1)$ such that $\mathbb{P}\left(\tau_{\infty} \leq T\right)>\epsilon$.

We define now a $C^{2}$-function $H: \mathbb{R}_{+}^{4} \rightarrow \mathbb{R}_{+}$by

$$
\begin{aligned}
H(S, I, C, V) & =\left(S-m-m \log \frac{S}{m}\right)+(I-1-\log I)+(C-1-\log C) \\
& +(V-1-\log V)
\end{aligned}
$$


where $m$ is a positive constant to be determined below.

Applying the infinitesimal operator $L$ on $H$, we obtain

$$
\begin{aligned}
L H(S, I, C, V)= & \left(1-\frac{m}{S}\right)\left(A-\beta(1-\rho) \frac{S I}{1+a I^{2}}+\eta I+\theta C-(\mu+\gamma) S+\varepsilon V\right) \\
& +\left(1-\frac{1}{I}\right)\left(\beta(1-\rho) \frac{S I}{1+a I^{2}}-\left(\mu+\delta_{1}+\eta+\alpha\right) I\right) \\
& +\left(1-\frac{1}{C}\right)\left(\alpha I-\left(\mu+\delta_{2}+\theta\right) C\right) \\
& +\left(1-\frac{1}{V}\right)(\gamma S-(\mu+\varepsilon) V) \\
& -\frac{\xi^{2} S^{2}}{2\left(1+a I^{2}\right)^{2}}-m \frac{\xi^{2} I^{2}}{2\left(1+a I^{2}\right)^{2}} \cdot \\
\leq & A-\left(\mu+\delta_{1}\right) I+m\left(\beta(1-\rho) \frac{I}{1+a I^{2}}+m(\mu+\gamma)+\mu+\delta_{1}+\eta+\alpha\right. \\
& +\mu+\delta_{2}+\theta+\mu+\varepsilon .
\end{aligned}
$$

Since $\frac{1}{1+a I^{2}}<1$ and choosing $m=\frac{\mu+\delta_{1}}{\beta(1-\rho)}$, we get

$$
L H(S, I, C, V) \leq A+m(\mu+\gamma)+\mu+\delta_{1}+\eta+\alpha+\mu+\delta_{2}+\theta+\mu+\varepsilon:=M>0 .
$$

Let $p \geq p_{0}$ and $T>0$ be arbitrary. Using Itô's formula [49], we obtain

$$
d H=L H d t+\frac{\xi}{1+a I^{2}}(m I-S) d W_{t}
$$

Therefore, we obtain

$$
d H \leq M d t+\frac{\xi}{1+a I^{2}}(m I-S) d W_{t}
$$

Integrating both sides of $(7)$ from 0 to $\tau_{p} \wedge T$, and then taking the expectation on both sides, we have

$$
0 \leq \mathbb{E} H\left(S\left(\tau_{p} \wedge T\right), I\left(\tau_{p} \wedge T\right), C\left(\tau_{p} \wedge T\right), V\left(\tau_{p} \wedge T\right)\right) \leq H(S(0), I(0), C(0), V(0))+M T .
$$

Define for each $h>0$

$$
\mathcal{H}(h):=\inf \left\{H(x), x_{i} \geq h \text { or } x_{i} \leq \frac{1}{h}, i=1,2,3,4\right\},
$$

where $x_{1}=S, x_{2}=I, x_{3}=C, x_{4}=V$ and we have $\lim _{h \rightarrow \infty} \mathcal{H}(h)=\infty$.

Therefore, letting $p \rightarrow \infty$ leads to

$$
\infty>H(S(0), I(0), C(0), V(0))+M T=\infty .
$$

This is a contradiction, so we have $\tau_{\infty}=\infty$, which completes the proof.

Remark 4. Let $N(t)=S(t)+I(t)+C(t)+V(t)$, according to system (2), we have

$$
\left.\left.\frac{d N(t)}{d t}=A-\mu N(t)-\delta_{1} I(t)-\delta_{2} C(t)\right) \leq \mu\left(\frac{A}{\mu}-N(t)\right)\right),
$$

then we have

$$
\frac{d\left(\frac{A}{\mu}-N(t)\right)}{d t}+\mu\left(\frac{A}{\mu}-N(t)\right) \geq 0
$$

Therefore

$$
\frac{A}{\mu}-N(t) \geq\left(\frac{A}{\mu}-N(0)\right) e^{-\mu t}
$$


if $\frac{A}{\mu}-N(0) \geq 0$, i.e., $S(0)+I(0)+C(0)+V(0) \leq \frac{A}{\mu}$, then

$$
S(t)+I(t)+C(t)+V(t) \leq \frac{A}{\mu}
$$

Thus the region

$$
\Delta=\left\{(S, I, C, V) \in \mathbb{R}_{+}^{4}: \mathbb{P}\left(S+I+C+V \leq \frac{A}{\mu}\right)=1\right\}
$$

is a positively invariant set of system (2).

In the rest of this paper, we always assume that $(S(0), I(0), C(0), V(0)) \in \Delta$

\subsection{Disease extinction}

In this subsection, we investigate the disease extinction according to the environmental noise intensity $\xi$. For simplicity, we consider the following notation:

$$
\langle f(t)\rangle=\frac{1}{t} \int_{0}^{t} f(u) d u
$$

Theorem 5. Let $(S(t), I(t), C(t), V(t))$ be the solution of system (2) with initial value $(S(0), I(0), C(0), V(0)) \in$ $\Delta$. If at least one of the following conditions hold

(i) $\xi^{2}>\frac{\beta^{2}(1-\rho)^{2}\left(1+a\left(\frac{A}{\mu}\right)^{2}\right)^{2}}{2\left(\mu+\delta_{1}+\alpha+\eta\right)}$ or

(ii) $\mathcal{R}_{s}<1$ and $\xi^{2} \leq \frac{\beta(1-\rho)\left(1+a\left(\frac{A}{\mu}\right)^{2}\right)}{S_{0}}$, where

$$
\mathcal{R}_{s}=\frac{\beta(1-\rho) S_{0}}{\mu+\delta_{1}+\alpha+\eta+\frac{\xi^{2} S_{0}^{2}}{2\left(1+a\left(\frac{A}{\mu}\right)^{2}\right)^{2}}} .
$$

Then

$$
\lim _{t \rightarrow \infty} \sup \frac{\log I(t)}{t}<0 \text { a.s. }
$$

namely $I(t)$ tends toward zero exponentially a.s., which means that the COVID-19 disease dies out with probability one. In addition, we have

$$
\lim _{t \rightarrow \infty} S(t)=S_{0}, \lim _{t \rightarrow \infty} I(t)=0, \lim _{t \rightarrow \infty} C(t)=0 \text { and } \lim _{t \rightarrow \infty} V(t)=V_{0} .
$$

Proof Using It $\tilde{\mathrm{A}}$ "s formula, we obtain

$$
d(\log I)=\left(\frac{S}{1+a I^{2}}-\left(\mu+\delta_{1}+\alpha+\eta\right)-\frac{\xi^{2} S^{2}}{2\left(1+a I^{2}\right)}\right) d t+\xi \frac{S}{1+a I^{2}} d W_{t}
$$

Integrating both sides of the equation (13) from 0 to $t$ and dividing by $t$, we get 


$$
\begin{aligned}
\frac{\log I(t)-\log I(0)}{t} & =\beta(1-\rho)\left\langle\frac{S}{1+a I^{2}}\right\rangle-\left(\mu+\delta_{1}+\alpha+\eta\right)-\left\langle\frac{\xi^{2} S^{2}}{2\left(1+a I^{2}\right)^{2}}\right\rangle \\
& +\frac{\xi}{t} \int_{0}^{t} \frac{S(u)}{1+a I(u)^{2}} d W_{u} \\
\leq \beta(1-\rho)\langle S(t)\rangle-\left(\mu+\delta_{1}+\alpha+\eta\right)-\frac{\xi^{2}}{2\left(1+a\left(\frac{A}{\mu}\right)^{2}\right)^{2}}\left\langle S(t)^{2}\right\rangle & \\
& +\frac{\xi}{t} \int_{0}^{t} \frac{S(u)}{1+a I(u)^{2}} d W_{u} \\
\leq \beta(1-\rho)\langle S(t)\rangle-\left(\mu+\delta_{1}+\alpha+\eta\right)-\frac{\xi^{2}}{2\left(1+a\left(\frac{A}{\mu}\right)^{2}\right)^{2}}\langle S(t)\rangle^{2} & \\
& +\frac{\xi}{t} \int_{0}^{t} \frac{S(u)}{1+a I(u)^{2}} d W_{u} .
\end{aligned}
$$

This leads to

$$
\begin{gathered}
\frac{\log I(t)}{t} \leq-\frac{\xi^{2}}{2\left(1+a\left(\frac{A}{\mu}\right)^{2}\right)^{2}}\left[\left(\langle S(t)\rangle-\frac{\beta(1-\rho)\left(1+a\left(\frac{A}{\mu}\right)^{2}\right)^{2}}{\xi^{2}}\right)^{2}\right. \\
\left.-\frac{\beta^{2}(1-\rho)^{2}\left(1+a\left(\frac{A}{\mu}\right)^{2}\right)^{4}}{\xi^{4}}\right]-\left(\mu+\delta_{1}+\alpha+\eta\right)+\phi(t) \\
\leq \frac{\beta^{2}(1-\rho)^{2}\left(1+a\left(\frac{A}{\mu}\right)^{2}\right)^{2}}{2 \xi^{2}}-\left(\mu+\delta_{1}+\alpha+\eta\right)+\phi(t), \\
\phi(t)=\frac{\log I(0)}{t}+\frac{\xi}{t} \int_{0}^{t} \frac{S(u)}{1+a I(u)^{2}} d W_{u} .
\end{gathered}
$$

By strong law of large numbers for local martingales [50] and taking the superior limit on the both sides of (15), we obtain

$$
\lim _{t \rightarrow \infty} \sup \frac{\log I(t)}{t} \leq \frac{\beta^{2}(1-\rho)^{2}\left(1+a\left(\frac{A}{\mu}\right)^{2}\right)^{2}}{2 \xi^{2}}-\left(\mu+\delta_{1}+\alpha+\eta\right) .
$$

If the condition $i$ ) is satisfied, then we have

$$
\lim _{t \rightarrow \infty} \sup \frac{\log I(t)}{t} \leq \frac{\beta^{2}(1-\rho)^{2}\left(1+a\left(\frac{A}{\mu}\right)^{2}\right)^{2}}{2 \xi^{2}}-\left(\mu+\delta_{1}+\alpha+\eta\right)<0 \text { a.s. }
$$


On the other hand, an integration of model (16) yields

$$
\left\{\begin{aligned}
\frac{S(t)-S(0)}{t} & =A-\beta(1-\rho)\left\langle\frac{S I}{1+a I^{2}}\right\rangle+\eta\langle I(t)\rangle+\theta\langle C(t)\rangle-(\mu+\gamma)\langle S\rangle+\varepsilon\langle V(t)\rangle \\
& -\frac{\xi}{t} \int_{0}^{t} \frac{S(u) I(u)}{1+a I(u)^{2}} d W_{u}, \\
\frac{I(t)-I(0)}{t} & =\beta(1-\rho)\left\langle\frac{S I}{1+a I^{2}}\right\rangle-\left(\mu+\delta_{1}+\alpha+\eta\right)\langle I\rangle+\frac{\xi}{t} \int_{0}^{t} \frac{S(u) I(u)}{1+a I(u)^{2}} d W_{u}, \\
\frac{C(t)-C(0)}{t} & =\alpha\langle I(t)\rangle-\left(\mu+\delta_{2}+\theta\right)\langle C(t)\rangle . \\
\frac{V(t)-V(0)}{t} & =\gamma\langle S(t)\rangle-(\mu+\varepsilon)\langle V(t)\rangle .
\end{aligned}\right.
$$

From system (16), we have

$$
\begin{gathered}
\langle C(t)\rangle=\frac{\alpha}{\mu+\delta_{2}+\theta}\langle I(t)\rangle-\frac{1}{\mu+\delta_{2}+\theta} \frac{C(t)-C(0)}{t}, \\
\langle V(t)\rangle=\frac{\gamma}{\mu+\varepsilon}\langle S(t)\rangle-\frac{1}{d+\varepsilon} \frac{V(t)-V(0)}{t}, \\
\frac{S(t)-S(0)}{t}+\frac{I(t)-I(0)}{t}+\frac{C(t)-C(0)}{t}+\frac{V(t)-V(0)}{t} \\
=A-\mu\langle S(t)\rangle-\left(\mu+\delta_{1}\right)\langle I(t)\rangle-\left(\mu+\delta_{2}\right)\langle C(t)\rangle-\mu\langle V(t)\rangle .
\end{gathered}
$$

Substituting (17) and (18) in (19), we obtain

$$
\langle S(t)\rangle=S_{0}-K\langle I(t)\rangle+\varphi(t),
$$

where

$$
\begin{gathered}
\varphi(t)=-\frac{(d+\varepsilon)}{\mu(\mu+\gamma+\varepsilon)}\left(\frac{S(t)-S(0)}{t}+\frac{I(t)-I(0)}{t}+\frac{C(t)-C(0)}{t}+\frac{V(t)-V(0)}{t}\right) \\
+\frac{(\mu+\varepsilon)\left(\mu+\delta_{2}\right)}{\mu(\mu+\gamma+\varepsilon)\left(\mu+\delta_{2}+\theta\right)}\left(\frac{C(t)-C(0)}{t}\right)+\frac{1}{\mu+\gamma+\varepsilon}\left(\frac{V(t)-V(0)}{t}\right) \\
K=\frac{(\mu+\varepsilon)\left(\left(\mu+\delta_{1}\right)\left(\mu+\delta_{2}+\theta\right)+\alpha\left(\mu+\delta_{2}\right)\right)}{\mu(\mu+\gamma+\varepsilon)\left(\mu+\delta_{2}+\theta\right)}
\end{gathered}
$$

Obviously $\varphi(t) \rightarrow 0$ as $t \rightarrow 0$. Substituting (20) in (14), we have

$$
\begin{aligned}
\frac{\log I(t)}{t} \leq & \beta(1-\rho)\left(S_{0}-K\langle I(t)\rangle+\varphi(t)\right)-\left(\mu+\delta_{1}+\alpha+\eta\right) \\
& -\frac{\xi^{2}}{2\left(1+a\left(\frac{A}{\mu}\right)^{2}\right)^{2}}\left(S_{0}-K\langle I(t)\rangle+\varphi(t)\right)^{2}+\frac{\xi}{t} \int_{0}^{t} \frac{S(u)}{1+a I(u)^{2}} d W_{u} \\
\leq & \left(\begin{array}{l}
\left.\mu+\delta_{1}+\alpha+\eta+\frac{\xi^{2} S_{0}^{2}}{2\left(1+a\left(\frac{A}{\mu}\right)^{2}\right)^{2}}\right)\left(\mathcal{R}_{s}-1\right) \\
\end{array}\right. \\
& -K\left(\begin{array}{l}
\left.\beta(1-\rho)-\frac{\xi^{2} S_{0}}{\left(1+a\left(\frac{A}{\mu}\right)^{2}\right)^{2}}\right)^{2} \\
(1+I)\rangle+\Psi(t),
\end{array}\right.
\end{aligned}
$$


where

$$
\begin{aligned}
\Psi(t)= & \frac{\xi}{t} \int_{0}^{t} \frac{S(u)}{1+a I(u)^{2}} d W_{u}+\beta(1-\rho) \varphi(t)+\frac{\log I(0)}{t} \\
& -\frac{\xi^{2}}{2\left(1+a\left(\frac{A}{\mu}\right)^{2}\right)^{2}}\left(\varphi(t)+2 S_{0}-2 K\langle I(t)\rangle\right) \varphi(t) .
\end{aligned}
$$

By strong law of large numbers for local martingales [50], we obtain

$$
\lim _{t \rightarrow \infty} \Psi(t)=0 \text { a.s. }
$$

If the condition $i i$ ) is satisfied, and by taking the superior limit on the both sides of (23), we obtain

$$
\lim _{t \rightarrow \infty} \sup \frac{\log I(t)}{t} \leq\left(\mu+\delta_{1}+\alpha+\eta+\frac{\xi^{2} S_{0}^{2}}{2\left(1+a\left(\frac{A}{\mu}\right)^{2}\right)^{2}}\right)\left(\mathcal{R}_{s}-1\right)<0 \text { a.s. }
$$

Equations (16) and (25) imply

$$
\lim _{t \rightarrow \infty} I(t)=0 \text { a.s. }
$$

According to the third equation of system (2), we can get the following solution

$$
C(t)=e^{-\left(\delta_{2}+\mu+\theta\right) t}\left(\int_{0}^{t} \alpha I(u) e^{-\left(\delta_{2}+\mu+\theta\right) u} d u+C(0)\right)
$$

Using the L'Hospital's rule and from (26), we obtain

$$
\lim _{t \rightarrow \infty} C(t)=\lim _{t \rightarrow \infty} \frac{\left(\int_{0}^{t} \alpha I(u) e^{-\left(\delta_{2}+\mu+\theta\right) u} d u+c_{0}\right)}{e^{\left(\delta_{2}+\mu+\theta\right) t}}=0 \text { a.s. }
$$

According to system (16), we have

$$
S(t)+I(t)+C(t)+V(t)=e^{-\mu t}\left(\int_{0}^{t}\left(A-\delta_{1} I(u)-\delta_{2} C(u)\right) e^{\mu u} d u+S(0)+I(0)+C(0)+V(0)\right)
$$

Applying L'Hospital's rule and according to (26) and (27), we get

$$
\lim _{t \rightarrow \infty}(S(t)+V(t))=\lim _{t \rightarrow \infty} \frac{\left(\int_{0}^{t}\left(A-\delta_{1} I(u)-\delta_{2} C(u)\right) e^{\mu u} d u+S(0)+I(0)+C(0)+V(0)\right)}{e^{\mu t}}=\frac{A}{\mu} \text { a.s. }
$$

Similarly from the fourth equation of system (2)

$$
\lim _{t \rightarrow \infty} V(t)=\lim _{t \rightarrow \infty} \frac{\left(\int_{0}^{t} \gamma S(u) e^{(\mu+\varepsilon) u}+V(0)\right)}{e^{(\mu+\varepsilon) t}}=\frac{\gamma}{\mu+\varepsilon} \times \lim _{t \rightarrow \infty} S(t) \text { a.s. }
$$

We thus obtain

$$
\begin{aligned}
& \lim _{t \rightarrow \infty} S(t)=\frac{A(\mu+\varepsilon)}{\mu(\mu+\varepsilon+\gamma)}=S_{0} \text { a.s. } \\
& \lim _{t \rightarrow \infty} V(t)=\frac{A \gamma}{\mu(\mu+\varepsilon+\gamma)}=V_{0} \text { a.s. }
\end{aligned}
$$

This completes the proof. 


\subsection{Persistence criteria}

This subsection is devoted to establish sufficient conditions for persistence in the mean of the disease of the stochastic system (2).

Definition 6. The model (2) is said to be persistent in mean, if

$$
\lim _{t \rightarrow \infty} \inf \langle I(t)\rangle>0,
$$

where $I(t)$ is the real infected population in the positive solution $(S(t), I(t), C(t), V(t))$ of system (2).

Theorem 7. For any initial value $(S(0), I(0), C(0), V(0)) \in \Delta$, if the following condition holds

$$
\xi^{2} \leq \frac{\beta(1-\rho)\left(1+a\left(\frac{A}{\mu}\right)^{2}\right)}{S_{0}} \text { and } \mathcal{R}_{s}^{\star}>1
$$

then

$$
I_{\mathrm{inf}} \leq \lim _{t \rightarrow \infty} \inf \langle I(t)\rangle \leq \lim _{t \rightarrow \infty} \sup \langle I(t)\rangle \leq I_{\text {sup }},
$$

where

$$
\begin{gathered}
I_{\mathrm{inf}}=\frac{K \beta(1-\rho)}{1+\left(\frac{A}{\mu}\right)^{2}}\left(\mathcal{R}_{s}^{\star}-1\right), \\
I_{\mathrm{sup}}=\frac{\left(\mu+\delta_{1}+\alpha+\eta+\frac{\xi^{2} S_{0}^{2}}{2\left(1+a\left(\frac{A}{\mu}\right)^{2}\right)^{2}}\right)}{K\left(\beta(1-\rho)-\frac{\xi^{2} S_{0}}{\left(1+a\left(\frac{A}{\mu}\right)^{2}\right)^{2}}\right)}\left(\mathcal{R}_{s}-1\right),
\end{gathered}
$$

and

$$
\mathcal{R}_{s}^{\star}=\frac{\beta(1-\rho) S_{0}}{\left(1+a\left(\frac{A}{\mu}\right)^{2}\right)\left(\mu+\delta_{1}+\alpha+\eta+\frac{\xi^{2}}{2}\left(\frac{A}{\mu}\right)^{2}\right)} .
$$

Proof From the last inequality of (23), we have

$$
\begin{aligned}
& \frac{\log I(t)}{t} \leq\left(\mu+\delta_{1}+\alpha+\eta+\frac{\xi^{2} S_{0}^{2}}{2\left(1+a\left(\frac{A}{\mu}\right)^{2}\right)^{2}}\right)\left(\mathcal{R}_{s}-1\right) \\
& -K\left(\beta(1-\rho)-\frac{\xi^{2} S_{0}}{\left(1+a\left(\frac{A}{\mu}\right)^{2}\right)^{2}}\right)\langle I(t)\rangle+\Psi(t),
\end{aligned}
$$

therefore

$$
\langle I(t)\rangle \leq \frac{\left(\mu+\delta_{1}+\alpha+\eta+\frac{\xi^{2} S_{0}^{2}}{2\left(1+a\left(\frac{A}{\mu}\right)^{2}\right)^{2}}\right)}{K\left(\beta(1-\rho)-\frac{\xi^{2} S_{0}}{\left(1+a\left(\frac{A}{\mu}\right)^{2}\right)^{2}}\right)}\left(\mathcal{R}_{s}-1\right)+\frac{\left(\Psi(t)+\frac{\log I(t)}{t}\right)}{K\left(\beta(1-\rho)-\frac{\xi^{2} S_{0}}{\left(1+a\left(\frac{A}{\mu}\right)^{2}\right)^{2}}\right)} .
$$

From (24) and for $\mathcal{R}_{s}>1$ and $\xi^{2} \leq \frac{\beta(1-\rho)\left(1+a\left(\frac{A}{\mu}\right)^{2}\right)}{S_{0}}$, we have

$$
\lim _{t \rightarrow \infty} \sup \langle I(t)\rangle \leq \frac{\left(\mu+\delta_{1}+\alpha+\eta+\frac{\xi^{2} S_{0}^{2}}{2\left(1+a\left(\frac{A}{\mu}\right)^{2}\right)^{2}}\right)}{K\left(\beta(1-\rho)-\frac{\xi^{2} S_{0}}{\left(1+a\left(\frac{A}{\mu}\right)^{2}\right)^{2}}\right)}\left(\mathcal{R}_{s}-1\right)=I_{\text {sup }}
$$




\begin{tabular}{llllll}
\hline Parameters & Fig. 2 & Fig. 3 & Fig. 4 & Fig. 5 & Fig. 6 \\
\hline \hline$A$ & 1 & 0.95 & 0.95 & 0.95 & 0.95 \\
\hline$\beta$ & 1 & 2.6 & 2.6 & 2.6 & 2.6 \\
\hline$\rho$ & 0.1 & 0 & 0 & 0 & - \\
\hline$a$ & 0.001 & 0.001 & 0.001 & 0.001 & 0.001 \\
\hline$\mu$ & 0.1 & 0.1 & 0.1 & 0.1 & 0.1 \\
\hline$\delta_{1}$ & 0.7 & 0.2 & 0.2 & 0.2 & 0.2 \\
\hline$\delta_{2}$ & 0.6 & 0.1 & 0.1 & 0.1 & 0.1 \\
\hline$\theta$ & 0.1 & 0.1 & 0.1 & 0.1 & 0.1 \\
\hline$\alpha$ & 0.6 & 0.6 & 0.6 & 0.6 & 0.6 \\
\hline$\gamma$ & 0.2 & 0.3 & 0.3 & 0.3 & 0.3 \\
\hline$\varepsilon$ & 0.6 & 0.6 & 0.6 & - & 0.6 \\
\hline$\xi$ & 0.01 & 0.01 & 0.01 & 0.01 & 0.01 \\
\hline
\end{tabular}

Table 1 The chosen parameters for the different numerical examples.

On the other hand, substituting (20) in (14) yields

$$
\begin{aligned}
& \frac{\log I(t)-\log I(0)}{t} \geq \frac{\beta(1-\rho)}{1+a\left(\frac{A}{\mu}\right)^{2}}\left(S_{0}-K\langle I(t)\rangle+\varphi(t)\right)-\left(\mu+\delta_{1}+\alpha+\eta\right) \\
& \quad-\frac{\xi^{2}}{2}\left(\frac{A}{\mu}\right)^{2}+\frac{\xi}{t} \int_{0}^{t} \frac{S(u)}{1+a I(u)^{2}} d W_{u} \\
&=\frac{\beta(1-\rho)}{1+a\left(\frac{A}{\mu}\right)^{2}} S_{0}-K \frac{\beta(1-\rho)}{1+a\left(\frac{A}{\mu}\right)^{2}}\langle I(t)\rangle+\frac{\beta(1-\rho)}{1+a\left(\frac{A}{\mu}\right)^{2}} \varphi(t) \\
&-\left(\mu+\delta_{1}+\alpha+\eta\right)-\frac{\xi^{2}}{2}\left(\frac{A}{\mu}\right)^{2}+\frac{\xi}{t} \int_{0}^{t} \frac{S(u)}{1+a I(u)^{2}} d W_{u} .
\end{aligned}
$$

Hence, by solving the inequality (35) and using the strong law of local martingales, we obtain

$$
\lim _{t \rightarrow \infty} \inf \langle I(t)\rangle \geq \frac{K \beta(1-\rho)}{1+\left(\frac{A}{\mu}\right)^{2}}\left(\mathcal{R}_{s}^{\star}-1\right)=I_{\text {inf }}
$$

Remark 8. It is easy to verify that $\mathcal{R}_{s}^{\star}<\mathcal{R}_{s}<R_{0}$.

\section{Numerical simulations}

This section is devoted to illustrate our theoretical findings by some numerical simulations. First, we will illustrate numerically the stochastic disease extinction. Next, we will give an example of numerical simulation for our problem persistence in mean. In addition, a comparison between our numerical results and COVID-19 clinical data will be given. This section will end by some numerical results illustrating the effect social distancing and vaccination on the disease spread. The chosen parameters of each numerical result are given in Table 1.

\subsection{The stochastic extinction}

In order to illustrate the stochastic disease extinction, we will use the parameters given in the second column of Table 1 . Within those parameters, we can easily calculate $\mathcal{R}_{s}=0.9959<1$ and we can easily verify that $\xi^{2} \leq \frac{\beta(1-\rho)\left(1+a\left(\frac{A}{\mu}\right)^{2}\right)}{S_{0}}$. According to Theorem 5, COVID-19 disease will die out stochastically. Now we will check this numerically; indeed, Fig. 2 shows the stochastic disease extinction for the same given parameters in the second column of Table 1. This is well observed from the stochastic trajectories since both the real and confirmed infected population vanish, while the susceptible and the vaccinated reach their maximal levels. It is interesting to notice that even if 

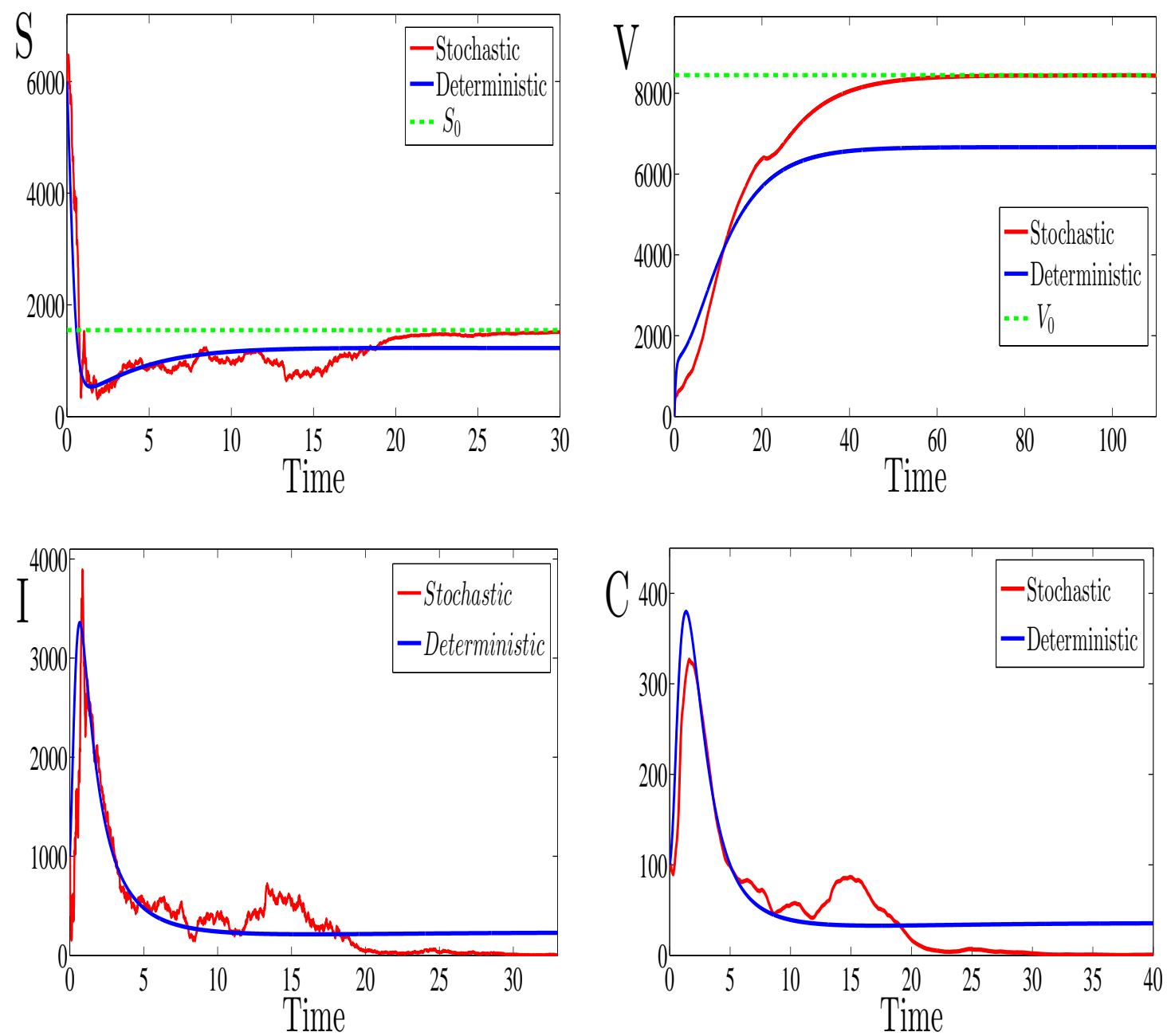

Fig. 2 The dynamical behavior of compartments $S, I, C$ and $V$ revealing the stochastic extinction of COVID-19 disease with $R_{0}=1.2676>1$ and $\mathcal{R}_{s}=0.9959<1$.

we have the stochastic extinction, the deterministic model will represent persistence of the disease, here we still have $R_{0}=1.2676>1$. Therefore, the stochastically modelling permits to have a wide view on the disease dynamics. Moreover, from this same figure, we can observe, that the stochastic solution converges toward the free-disease steady state $E_{f}$.

\subsection{Persistence of the disease}

In order to check the stochastic persistence of the disease, we will use the parameters given in the third column of Table 1 . Within those parameters, we can easily calculate $\mathcal{R}_{s}^{\star}=1.0639>1$ and we can easily verify that $\xi^{2} \leq \frac{\beta(1-\rho)\left(1+a\left(\frac{A}{\mu}\right)^{2}\right)}{S_{0}}$. Therefore, from Theorem 7 , we expect that COVID19 disease will persist. Now we will check this numerically; indeed, Fig. 2 show the time evolution of our SICV four compartments model for the same Table 1 third column parameters. From this figure, we observe that the trajectories representing real and confirmed infected individuals, for both deterministic and stochastic cases, remain at a strictly positive level which means that the disease persist. In addition, the plotted curves show the stochastic solution fluctuate around the deterministic one. 

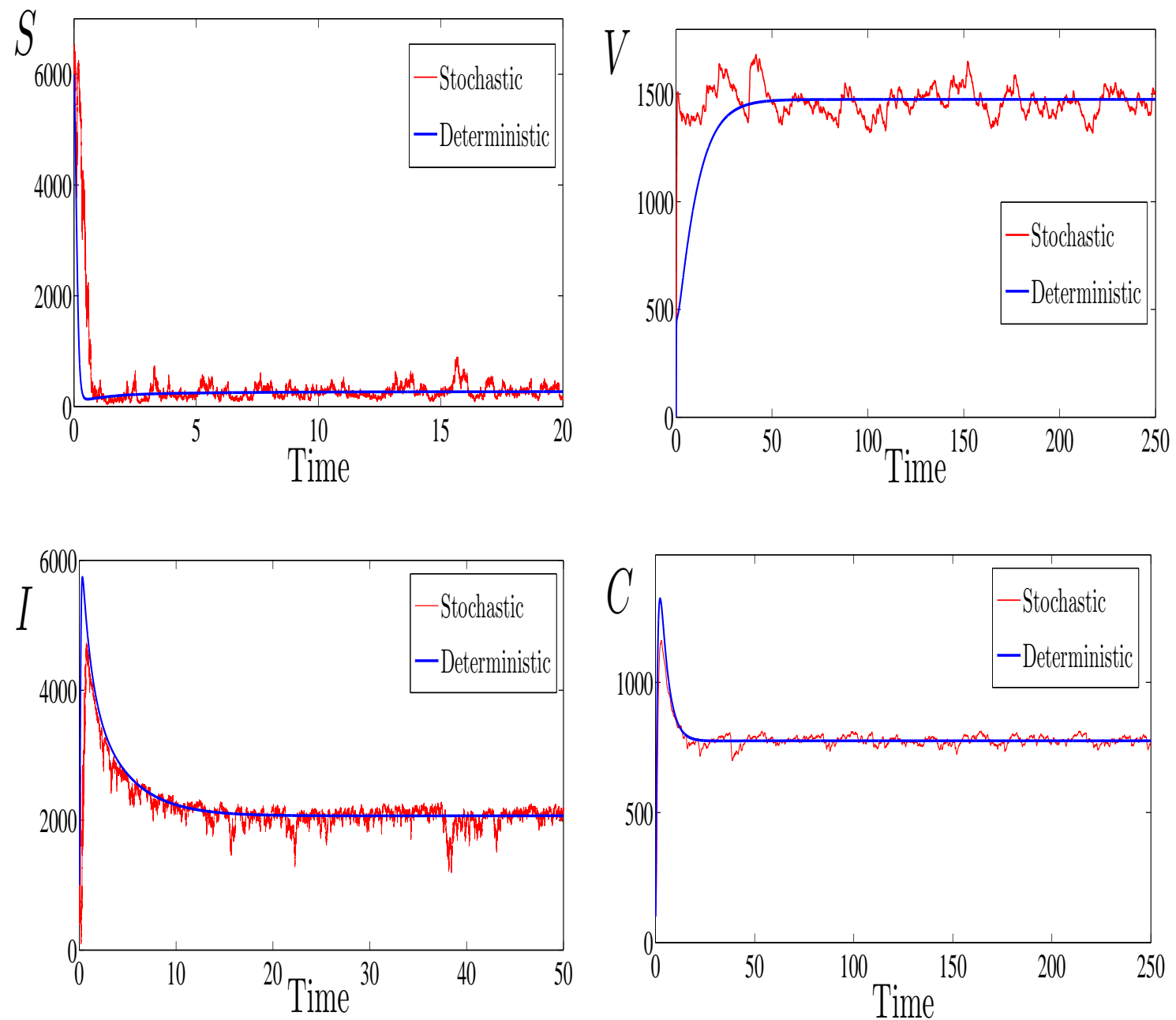

Fig. 3 The dynamical behavior of compartments $S, I, C$ and $V$ revealing the persistence of COVID-19 disease for stochastic and deterministic cases with $\mathcal{R}_{s}^{\star}=1.0639>1$

\subsection{Application to Morocco COVID-19 clinical data}

We have chosen to make our comparison, the Moroccan clinical data during the period between October 18, 2020 and March 27, 2021 [51, 52]. Figure 4 shows the time evolution of infected cases, we observe a significant good approach between the curves representing the model numerical results and the clinical data. Hence, our model have shown its efficiency in approaching and predicting the second wave of COVID-19 pandemic. Moreover, the stochastic curve shows good approximation to the clinical data than the deterministic plot. 


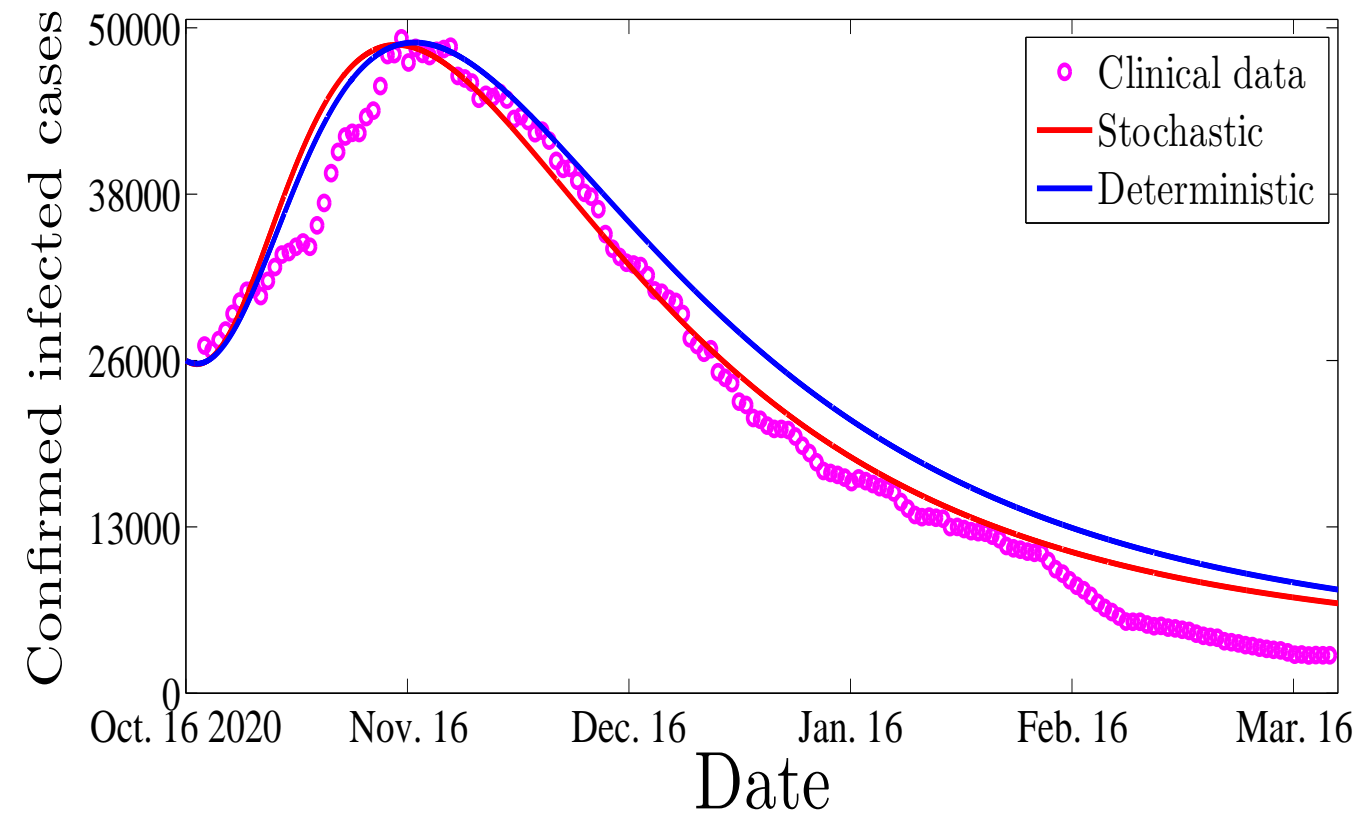

Fig. 4 The time evolution of confirmed infected cases, the stochastic trajectory (red color), the deterministic trajectory (blue color). The clinical infected cases are illustrated by magenta circles.

\subsection{The effect of the vaccination strategy on COVID-19 pandemic spread}

In this subsection, we will study the effect of vaccination strategy in fighting against the spread of COVID-19 pandemic.

Figure 5 shows the time evolution of the real infected cases for the parameters given in the fifth column of Table 1. The left-hand side of this figure depicts the dynamics for the stochastic model, while the right-hand side illustrates the dynamics for the deterministic model. For both cases, we can easily observe the effect of vaccination strategy on reducing the spread of COVID-19 infection. Indeed, by increasing the vaccination rate a significant reduce of the real infected individuals is observed which clearly reveals the role of the vaccination strategy in reducing the infection severity.
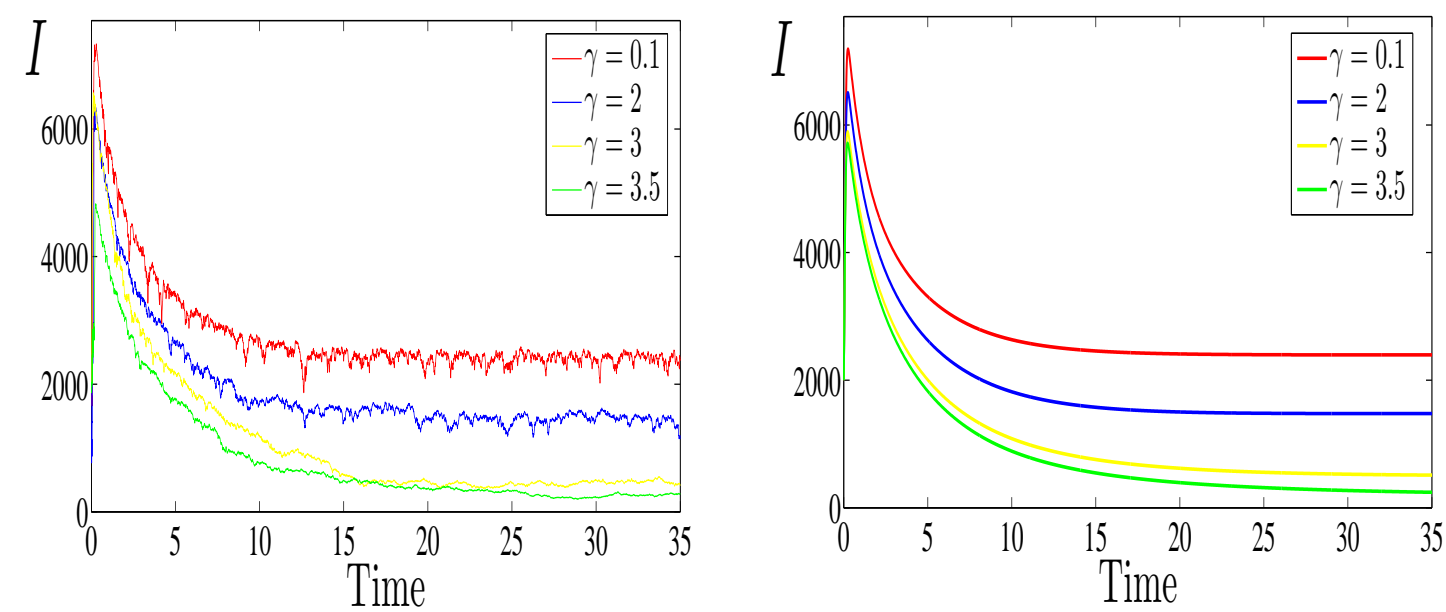

Fig. 5 The dynamical behavior of real infected cases for the stochastic model (left) and for the deterministic model (right) revealing the effect of vaccination rate on COVID-19 pandemic model. 

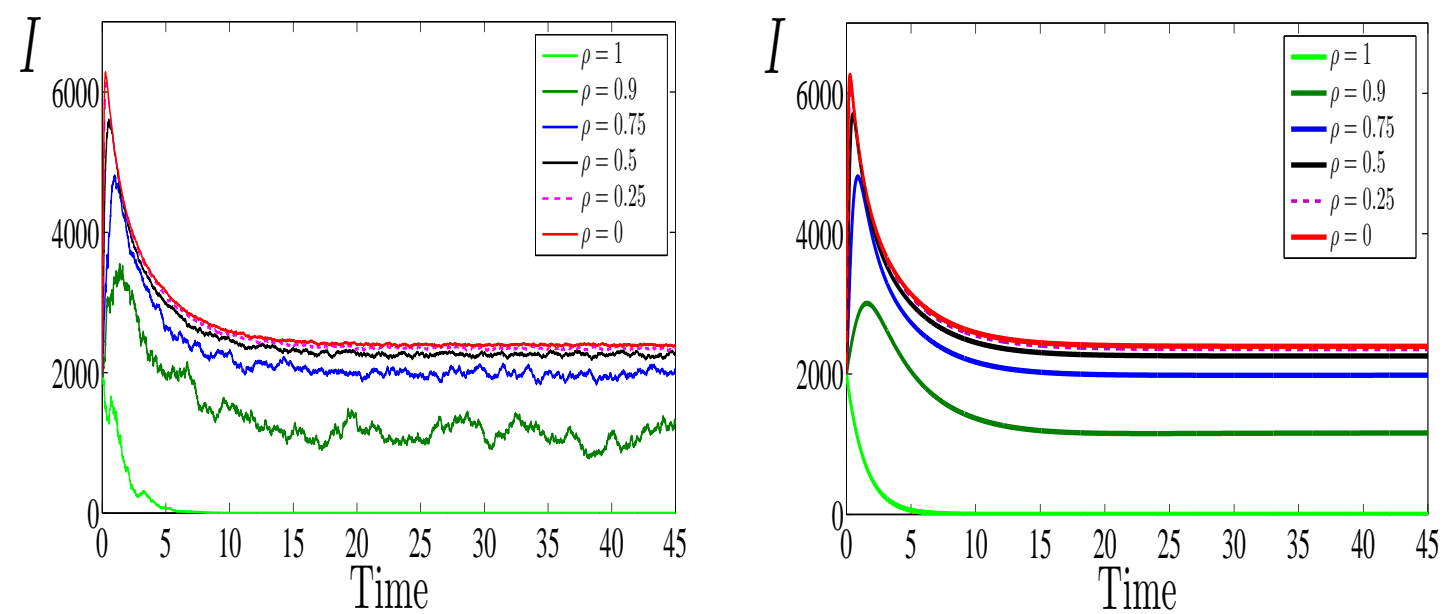

Fig. 6 The dynamical behavior of real infected cases for the stochastic model (left) and for the deterministic model (right) revealing the effect of social distancing effect on COVID-19 pandemic model.

\subsection{The effect of social distancing strategy on the spread of the COVID-19 pandemic}

Figure 6 shows the time evolution of real infected cases for different values of parameter reflecting the efficiency of social distancing effect. The plots concerning the stochastic model are represented in left-hand side of the figure; while the obtained curves from the deterministic model are drawn in the right-hand side. We observe that for the two cases, both stochastic and deterministic, the importance of social distancing in reducing the infection spread. More precisely, we remark that when the social distancing is well respected the number of COVID-19 infected individuals is reduced significantly.

\section{Conclusion}

The COVID-19 pandemic caused by the novel SARS-COV-2 virus, which first appeared in China in December 2019, has so far posed a serious threat to global public health. Doctors and scientific researchers are working day and night to find an adequate vaccine in the hope of eradicating this pandemic and returning populations to normal life before COVID-19. In this paper, we have studied a stochastic and deterministic novel model describing the spread of the COVID-19 pandemic with a vaccination strategy and crowding effect. The model consisted of four compartments, namely, the susceptible $S$, the real infected $I$, the confirmed infected $C$ and the vaccinated individuals $V$. First, we have studied the local stability of the equilibrium points of the corresponding deterministic model. Next, we have proved that the stochastic model has a positive unique solution as this is essential in any population dynamics. Then we have given sufficient conditions for the stochastic extinction and the persistence in mean of the disease. Finally, we have confirmed our theoretical results by adequate numerical simulations. The proposed model was supported by a comparison with real Moroccan clinical data on Covid-19 between October 18, 2020 and March 27, 2021. It was revealed that for stochastic and deterministic cases, vaccination strategy plays an essential role in controlling COVID-19 spread. In addition, it was shown that when the social distancing is well respected the number infected individuals will be reduced considerably. We can conclude from our study that the proposed model may be conductive to understand the role of some control strategies such as vaccination and social distancing in order to prevent the COVID-19 disease from spreading extensively in the population. 


\section{Compliance with ethical standards}

Conflict of interest The authors declare that they have no conflict of interest.

\section{Funding}

The authors declare that no funds, grants, or other support were received during the preparation of this manuscript.

\section{Data Availability}

The data sets that support the theoretical results of this study are available on the official website of the Moroccan Ministry of Health on COVID-19 or the official website of the World Health Organization (WHO):

https://www.who.int/countries/mar/

https://www.sante.gov.ma/

\section{References}

1. Kermack, W.O., McKendrick, A.G.: A contribution to the mathematical theory of epidemics. Proc. R Soc. Lond. A. 115, 700-721(1927)

2. Anderson, Roy, M., May, R.M.: Population biology of infectious diseases : Part I. Nature 280, 361-367 (1979)

3. Hethcote, Herbert, W.: The mathematics of infectious diseases. SIAM rev. 42, 599-653 (2000)

4. Xiao, D., Ruan, S.: Global analysis of an epidemic model with nonmonotone incidence rate, Math. Biosci. 208, 419-429 (2007)

5. Wang, J.J., Zhang, J.Z., Jin, Z.: Analysis of an SIR model with bilinear incidence rate, Nonlinear Anal: Real World Appl. 11, 2390-2402 (2010)

6. Capasso, V., Serio, G.: A generalization of the kermack-mckendrick deterministic epidemic model, Math. Biosci. 42, 43-61 (1978)

7. Nakata, Y., Kuniya, T.: Global dynamics of a class of SEIRS epidemic models in a periodic environment, J. Math. Anal. Appl. 363, 230-237 (2010)

8. Liu, W.M., Levin, S.A., Iwasa, Y.: Influence of nonlinear incidence rates upon the behavior of SIRS epidemiological models, J. Math. Biol. 23, 187-204 (1986)

9. Chen, Y.C., Lu, P.E., Chang, C.S., Liu, T.H.: A time-dependent SIR model for COVID-19 with undetectable infected persons, IEEE Trans. Net. Sci. Eng. 7, 3279-3294 (2020)

10. Cooper, I., Mondal, A., Antonopoulos, C.G.: A SIR model assumption for the spread of COVID-19 in different communities, Chaos Sol. Frac. 139, 110057 (2020)

11. Volpert, V., Banerjee, M., Petrovskii, S.: On a quarantine model of coronavirus infection and data analysis, Math. Mod. Nat. Phen. 1524 (2020)

12. Zhao, S., Chen, H.: Modeling the epidemic dynamics and control of COVID-19 outbreak in China, Quant. Biol. 8 11-19 (2020)

13. Ullah, S., Khan, M.A.: Modeling the impact of non-pharmaceutical interventions on the dynamics of novel coronavirus with optimal control analysis with a case study, Chaos Sol. Frac. 139110075 (2020)

14. Ali, A., Alshammari, F.S., Islam, S., Khan, M.A., Ullah, S.: Modeling and analysis of the dynamics of novel coronavirus (COVID-19) with Caputo fractional derivative, Resul. Phys. 20 103669 (2021)

15. Irwin, A.: What it will take to vaccinate the world against COVID-19, Nature 592 176-178 (2021)

16. Upadhyay, R.K., Chatterjee, S., Saha, S., Azad, R.K.: Age-group-targeted testing for COVID19 as a new prevention strategy. Nonlinear Dynamics 101 1921-1932 (2020)

17. Malik, A., Khursheed, A., Nitendra, K.: COEFFICIENT IDENTIFICATION IN SIQR MODEL OF INVERSE PROBLEM OF COVID-19. Euro. J. Molec. Clin. Med. 7, 2515-8260 (2020)

18. Odagaki, T.: Analysis of the outbreak of COVID-19 in Japan by SIQR model, Inf. Dis. Model. 5, 691-698 (2020) 
19. Crokidakis, N.: Modeling the early evolution of the COVID-19 in Brazil: Results from a SIQR model, Inter. J. Modern. Phy. C. 31, 1-7 (2020)

20. Odagaki, T.: Exact Properties of SIQR model for COVID-19. Physica A 564(C), 125564 (2021)

21. Khyar, O., Allali, K.: Global dynamics of a multi-strain SEIR epidemic model with general incidence rates: application to COVID-19 pandemic, Nonl. Dyn. 102, 489-509 (2020)

22. Artiono, R., Prawoto, B.P.: The effects of vaccination to the dynamics of rubella virus with seasonality, Commun. Math. Biol. Neurosci. Article ID 9 (2020)

23. Li, B., Qin, C., Wang, X.: Analysis of an SIRS epidemic model with nonlinear incidence and vaccination, Commun. Math. Biol. Neurosci. Article ID 2 (2020)

24. Liu, Q., Jiang, D., Hayat, T., Alsaedi, A.: Threshold behavior in a stochastic delayed SIS epidemic model with vaccination and double diseases, J. Frank. Ins. 356, 7466-7485 (2019)

25. Liu, Q., Jiang, D.: The threshold of a stochastic delayed SIR epidemic model with vaccination, Physica A 461, 140-147 (2016)

26. Zhang, X., Jiang, D., Hayat, T., Ahmad, B.: Dynamical behavior of a stochastic SVIR epidemic model with vaccination, Physica A 483, 94-108 (2017)

27. Akdim, K., Ez-zetouni, A., Zahid, M.: A stochastic vaccinated epidemic model incorporating Lévy processes with a general awareness-induced incidence, Inter. J. Biomath. (2021).

28. Mahrouf, M., Lotfi, E.M., Hattaf, K., Yousfi, N.: Non-Pharmaceutical Interventions and Vaccination Controls in a Stochastic SIVR Epidemic Model, Differ. Eq. Dyn. Sys. (2020).

29. Akdim, K., Ez-zetouni, A., Danane, J., Allali, K.: Stochastic viral infection model with lytic and nonlytic immune responses driven by LÃC) vy noise. Physica A 549, 124367 (2020)

30. Rajaji, R., Pitchaimani, M.: Analysis of stochastic viral infection model with lytic and nonlytic immune responses, Stochastic Analysis and Applications 1-16 (2019)

31. Mao, X., Marion, G., Renshaw, E.: Environmental Brownian noise suppresses explosions in population dynamics, Stoch. Proc. Appl. 97 (1), 95-110 (2002)

32. Khan, T., Khan, A., Zaman, G.: The extinction and persistence of the stochastic hepatitis B epidemic model, Chaos Sol. Frac. 108, 123-128 (2018)

33. Ji, C., Jiang, D.: Threshold behaviour of a stochastic SIR model, Appl. Math. Model. 38, 5067-5079 (2014)

34. Liu, X., Yang, L.: Stability analysis of an SEIQV epidemic model with saturated incidence rate, Nonl. Ana.: Real World Appl. 13, 2671-2679 (2012)

35. Hattaf, K., Mahrouf, M., Adnani, J., Yousfi, N.: Qualitative analysis of a stochastic epidemic model with specific functional response and temporary immunity, Physica A 490, 591-600 (2018)

36. Zhao, Y., Jiang, D.: The threshold of a stochastic SIRS epidemic model with saturated incidence, Appl. Math. Lett. 34, 90-93 (2014)

37. Liu, X.Q., Zhong, S.M., Tian, B.D., Zheng, F.X.: Asymptotic properties of a stochastic predator-prey model with Crowley-Martin functional response, J. Appl. Math. Comp. 43, 479-490 (2013)

38. Ji, C., Jiang, D., Yang, Q., Shi, N.: Dynamics of a multigroup SIR epidemic model with stochastic perturbation, Automatica 48, 121-131 (2012)

39. Cao, B., Shan, M., Zhang, Q., Wang, W.: A stochastic SIS epidemic model with vaccination, Physica A 486, 127-143 (2017)

40. Zhao, Y., Jiang, D.: The threshold of a stochastic SIS epidemic model with vaccination, Appl. Math. Comp. 243, 718-727 (2014)

41. El Koufi, A., Adnani, J., Bennar, A., Yousfi, N.: Analysis of a stochastic SIR model with vaccination and nonlinear incidence rate, Inter. J. Differ. Equ. 1-9 (2019)

42. Meng, X., Chen, L.: The dynamics of a new SIR epidemic model concerning pulse vaccination strategy, Appl. Math. Comp. 197, 582-597 (2008)

43. Boukanjime, B., Caraballo, T., El Fatini, M., El Khalifi, M.: Dynamics of a stochastic coronavirus (COVID-19) epidemic model with Markovian switching, Chaos Sol. Frac. 141, 110361 (2020)

44. Adak, D., Majumder, A., Bairagi, N.: Mathematical perspective of COVID-19 pandemic: disease extinction criteria in deterministic and stochastic models. Chaos Sol. Frac. 142, 110381 (2021)

45. He, S., Tang, S., Rong, L.: A discrete stochastic model of the COVID-19 outbreak: Forecast and control, Math. Biosci. Eng. 17, 2792-2804 (2020) 
46. Rihan, F.A., Alsakaji, H.J., Rajivganthi, C.: Stochastic SIRC epidemic model with time-delay for COVID-19, Adv. Differ. Equ. 2020, 1-20 (2020)

47. Danane, J., Allali, K., Hammouch, Z., Nisar, K.S.: Mathematical analysis and simulation of a stochastic COVID-19 Levy jump model with isolation strategy, Resul. Phy. 23, 103994 (2021)

48. Diekmann, O., Heesterbeek, J.A.P., Metz, J.A.J.: On the definition and the computation of the basic reproduction ratio $R_{0}$ in the models for infectious disease in heterogeneous populations, J. Math. Biol. 28, 365 (1990)

49. Mao, X.: Stochastic Differential Equations and Applications, 2nd Edition (Horwood, 2008).

50. Liptser, R.S.: A strong law of large numbers for local martingales, Stochastics 3, 217-228 (1980)

51. Statistics of World Health Organization (WHO) https://www.who.int/countries/mar/.

52. Statistics of Moroccan health ministry on COVID-19, https://www.sante.gov.ma/. 